\title{
ASSOCIATION BETWEEN JUVENILE SYSTEMIC LUPUS ERYTHEMATOSUS AND RENAL THROMBOTIC MICROANGIOPATHY: CASE REPORT
}

Mikael Albuquerque do Bú (Universidade Federal de Campina Grande (UFCG), Campina Grande, PB, Brasil), Aline Cordeiro de Azevêdo (Universidade Federal de Campina Grande (UFCG), Campina Grande, PB, Brasil), Ana Amélia Soares de Lima (Universidade Federal de Campina Grande (UFCG), Campina Grande, PB, Brasil), Juliana Clemente do Rêgo (Universidade Federal de Campina Grande (UFCG), Campina Grande, PB, Brasil), Lucas de Moura Brito (Universidade Federal de Campina Grande (UFCG), Campina Grande, PB, Brasil), Rennan Loureiro Batista Marinho (Universidade Federal de Campina Grande (UFCG), Campina Grande, PB, Brasil), Sofia Nunes Pinto de Oliveira (Universidade Federal de Campina Grande (UFCG), Campina Grande, PB, Brasil), Wilker John Barreto (Universidade Federal de Campina Grande (UFCG), Campina Grande, PB, Brasil), Marcus Ivanovith Fernandes (Universidade Federal de Campina Grande (UFCG), Campina Grande, PB, Brasil), Evânia Claudino Queiroga de Figueiredo (Universidade Federal de Campina Grande (UFCG), Campina Grande, PB, Brasil)

\section{BACKGROUND}

Thrombotic microangiopathy (TMA) is characterized by a clinical presentation composed by thrombocytopenia, microangiopathic hemolytic anemia and organ damage. It may be manifested through several presentations, including acute kidney injury, which is a common feature due to the apparent propensity of the glomerular circulation to endothelial damage and occlusion. Regarding systemic lupus erythematosus, thrombotic microangiopathy is reported in $8 \%-15 \%$ of the biopsies performed in patients with this autoimmune disease. The pathogenic mechanisms related to this specific situation are still unknown and the treatment to be performed is the one specific to lupus.

\section{CASE REPORT}

Female patient, 12 years old, previously diagnosed with systemic lupus erythematosus. Besides this diagnosis, she was affected by systemic arterial hypertension. The patient was admitted with symptoms as malar rash, low back pain, and edema of lower limbs and face. At the moment, she was being treated with azathioprine, captopril, amlodipine besylate, furosemide, hydroxychloroquine sulfate, and calcium carbonate and vitamin D mineral supplement. At physical examination, the lower limbs edema was soft and without phlogistic signs. It was also noticed the presence of purpura and ecchymoses in the lower limbs. Laboratory tests revealed proteinuria, anemia, leucopenia and high levels of creatinine and urea. Urinalysis showed the presence of proteins, red blood cells (10 per field) and some bacteria. The laboratory tests corresponding to the research for antiphospholipid antibody syndrome presented negative results. Renal biopsy revealed diffuse proliferative glomerulonephritis, with increasing (11 in 30 glomeruli), and with a slight tubulointerstitial repercussion; in addition, presented associated thrombotic microangiopathy. Abdominal ultrasound exam revealed small-volume ascites and small-sized ovaries. The recommended initial course to this case was the patient's hospitalization, with pulse therapy with Cyclophosphamide. Due to the fact that her disease was refractory to this treatment, the use of mycophenolate mofetil was started.

\section{CONCLUSION}

Renal involvement is present in many patients with systemic lupus erythematosus, being one of the main predictors for mortality. In the case described, the finding of thrombotic microangiopathy translates into the presence of fibrin thrombi in the glomerular arterioles and capillaries. As renal repercussions of thrombotic microangiopathy, the patient presented diffuse proliferative glomerulonephritis on renal biopsy. This result was consistent both with the urinalysis and with the patient's clinical condition, represented by lower limb edema and hypertension. 